\title{
Meaningful Practice Intervention: What Does It Look Like?
}

\author{
Brian Fairman* Adam Voak Hurriyet Babacan \\ Cairns Institute, James Cook University McGregor Rd. Cairns, Queensland 4878, Australia. \\ *E-mail of the corresponding author: Brian.Fairman@jcu.edu.au
}

\begin{abstract}
This paper seeks to investigate critically the appropriateness of the use of common everyday language expressions such as 'of course', and consider the possible effects of our acceptance of these 'common sense truths' regarding the way things ought to be, without pausing to question their legitimacy. The background to this paper stems from the work the authors have undertaken in developing national education systems. We suggest that acknowledging our implicit 'ideological' frame of reference may significantly assist Vocational training practitioners to challenge the 'hidden' developmental context for the prescribed training practices and interventions, rather than simply continuing to perpetuate the 'way things are', our observations of this behavior in development contexts has informed the direction of this paper. In the rapidly changing educational environment, there is a need to at least confront existing practices/systems in order to ensure that training outcomes fit issues that are currently relevant to the community, and are not merely perpetuating previously 'dominant values' which are no longer appropriate. We are concerned that, despite the difficulty and personal challenges involved in posing interrogative questions of human development program implementation and standard designed 'interventions', if these questions remain unchallenged, the dominant values are likely to endure, a consideration of utmost importance. This paper explores the implementation of a balanced meaningful practice intervention model based on the assumption that a balanced cross-culturally relevant training approach requires the application of an appropriate model of planned intervention. Such an approach will require the acknowledgement that the asking of difficult and confronting questions around existing 'institutionalized practice' are essential if meaningful and relevant change practices are to be instituted.
\end{abstract}

Keywords: Meaningful practice, Applied learning, Vocational education and training, Vocational imperialism.

DOI: $10.7176 /$ RHSS/11-22-01

Publication date: November $30^{\text {th }} 2021$

\section{Introduction}

The discipline behind Applied Learning involves engaging in meaningful and authentic learning experiences, where learning activities are structured to encourage and support learners to think, collaborate, communicate and engage positively with the experienced world around them (Barron \& Darling-Hammond, 2008). Applied Learning activities involve creative projects, independent and directed research, or simulated real-world situations, and these should be directly informed by a real-world context allowing the strategic application of meaningful skills, theories and models. Pivotal to the successful application of an Applied Learning process, is for the learners to immerse themselves in critical reflection regarding their practice so as to be able to: (i) cement their learning experiences; (ii) enhance the application of learned techniques; (iii) transfer the retention of learned approaches; and (iv) sharpen their powers of recall (Stanchfield, 2013). A critical issue in this respect is that learning should be transformative rather than transactional. Transformative learning is a major theory of adult education which derives from the view that education is not neutral (Freire, 1970; Mezirow, 2000), implying that it can either (i) reproduce the status quo or (ii) be emancipatory by encouraging learners to question, and transform the knowledge being discussed (Freire \& Shor, 1986). The transformative learning process involves transforming 'frames of reference' through: (i) critical reflection on the relevance of key material; (ii) evaluation of contested beliefs through meaningful dialogue; (iii) taking action based on the outcomes of one's reflective insight, and (iv) critically assessing the action taken (Babacan \& Babacan, $2015,2018)$.

\section{Frames of Reference}

It has been noted that the modern capitalist system organizes and redefines societal arrangements through the control of production, consumption, regulation of labor mobility and residential arrangements (Robinson et al., 2004). Within this system, globalization is the 'global enmeshment' of money, people, ideas, images, values and technologies that flow in swift and defining manner across the world (Beck, 2000). Global processes operate across borders and, as a consequence, act to diminish national and cultural identities. An additional complexity regarding this procedural framework is that the processes of change are not even. Hallinger (1998) noted that the social, economic, political and cultural institutions face the challenge of adapting to the same global standards in trade, commerce, education, human rights and industry, but they have different social, financial and political contexts. In this respect, Olaniran and Agnello (2008) opined that globalisation and global education pose major challenges to national and social equity through the perpetuation of educational hegemony. These authors define hegemony, as used in this discussion, as the imposition of a dominant culture on a non-dominant group (Olaniran 
\& Agnello, 2008). Given this external imposition of structure, the result of this dominance is not only economic reform but educational restructuring, and is constructed by the more 'developed' nations in their interest placing a central focus on their economic and market needs. Western and neo-liberal forms of education, which are widely accepted as dominant forms of education, have an imperialist/neo-colonial attitude, which has consistently failed to enhance Indigenous practices (Alphonse et al., 2008). These neo-colonial processes are seen as having inbuilt prejudices, including distributive injustices (related to economic and social capital), procedural injustices (denial of rights and representation, plus exclusion from decision making) and recognitive injustices (denial of the equality of social and cultural identities (Deutsch, 2006; Gale \& Densmore, 2000). These central and defining processes are activated through; (i) dominant theoretical and practice-based constructs, values and norms, (ii) the use of Eurocentric epistemology, and (iii) the shaping use of language which underpins the curriculum and teaching practice (Noble, 2004). It is argued that this hegemonic approach hides many tensions, resulting in manifestly undemocratic impacts for teachers, learners and minority groups in many countries (Olaniran \& Agnello, 2008). Nguyen et al. (2015) have identified six social and cultural points of tension in international collaborations relevant to educational contexts, and these include: (i) Westernizing and universalizing basic educational issues versus their Indigenization and authentization; (ii) lacking balance between local and international perspectives; (iii) overlooking differing perceptions of need; (iv) a tension in imposing external dominant culture whilst avoiding respect for alternative culture and customs; (v) misrepresenting context appropriateness; and (vi) ignoring power differentials between local and international partners. Farmer et al (2020) identify that interventions need to be responsive to dynamic cultural, developmental and ecological factors and there is a need for heterogeneity of responses in the face if difference.

Against this background, the intent of this paper is to provide a more equitable framework for Vocational Educational practitioners which will allow them to design more meaningful approaches and policy directions for training program interventions in specific environments. In particular, it will address the issue of the application of 'current regional' models of Vocational Education and Training (VET) interventions which could be viewed through the critical lens of 'cultural imperialism' (Allais, 2014). In this respect, we suggest that it is time for VET practitioners to re-examine their modus operandi by (i) questioning and challenging their historical perspectives, and (ii) examining their adherence to Eurocentric vocational education and training practices. This critical review should be couched in terms of the relevance and applicability of the educational practices to an ASEAN milieu. In this respect, we have examined current planned interventions by Australian practitioners in the ASEAN VET arena, in particular in several regions in Indonesia, and we acknowledge that the socio-economic challenges in each context are subtly different. We also recognized that a well-intentioned but ultimately miscued planned intervention, may leave attempts to improve practice in an ASEAN region open to challenges of using external influence for external gain. It is therefore absolutely essential that the selected 'models' are transparently seen as being applicable to an individual country and regional circumstances, respecting, honouring and acknowledging the prevailing social and cultural values.

In 2019, the President of the Republic of Indonesia, Joko Widodo, announced the recognition of the importance of education, particularly noting a focus on the VET sector. This is to be geared to employment outcomes and for preparing Indonesians for the new and emerging areas of the economy which are being prioritised by the government (Dayaram et al., 2020). The Minister of Education and Culture, Nadiem Makarim, in detailing the new Kampus Merdeka policy, describes this industry competency development as requiring conscientious academic and industry engagement, suggesting that 'Simulating real industry problems, [and] working together with industry professionals is going to be one of the best preparations to combine academic learning in the university with industry'(Neumann, 2021). This significant policy shift in applied learning and vocational education in Indonesia presents many challenges for educational institutes, vocational providers and indeed companies wishing to provide educational services to their staff.

The encouraging of recipient-country ownership of training programs is, we suggest, most appropriately engineered through transparent local engagement, and this can be most easily achieved through the piloting of planned training interventions which have 'shared ownership models' of delivery. During this piloting stage, reflection on the process by participants immersed in the local culture is an essential activity (Lawler \& Sillitoe, 2013). As noted by Fairman et al. (2020) 'The Malang Declaration', openly collaborating with international facilitators and encouraging models of sharing information amongst key stakeholders, will go a considerable way towards ensuring introduced training interventions and actually empower inclusiveness practices by being culturally sensitive (Fairman et al., 2020).

\section{The Cultural Lens}

An examination of the cross-cultural issues in planned training interventions may reveal the unintentional and unsuspected underpinning 'cultural' biases of many mainstream vocational training interventions perpetuated by institutional practice and professional practitioners (Gopalkrishnan \& Babacan, 2015). In this respect, it is reasonable to consider carefully how one might view the nature and extent of this problem, and to ask whether it 
depends upon the self-reflective position that the presenter holds. Experience has shown that whilst some practitioners may be clear about their ideological positions and rationale for engaging in vocational skills development practice, others are patently less so (Fairman, 2018; McIntosh, 2015). Stuart Hall 1983, in a presentation to a Melbourne University audience, noted that when we declare 'of course' in our common everyday speech, we are being at our most ideological:

The moment you say 'of course' to an ideology, you are in the most ideological bit of it, and the only way of coming out of it is to say not 'of course' at all but 'why of course'(Hall, 1983)

Hall's approach to examining one's own 'motives' is applicable in everyday life circumstances as well as vocational training interventions, since it demands a reflective and thoughtful examination of 'why of course'. Whilst in our own cultures, with our own ideologies, things 'naturally' make sense, but Fairman et al. (2020) have explored these perspectives in relation to the development of the 'Malang Declaration' (Fairman et al., 2020).

It is our contention that by applying a theoretical framework of cultural studies to the examination and implementation of applied learning methodologies will provide a countervailing context to the hidden language and cultural assumptions behind program design. This can be illustrated when we juxtapose these 'ways of thinking' and 'ways of seeing' about our world, onto (i) the wider ASEAN circumstances, (ii) the differences in various ASEAN Nation States and (iii) specific Indonesian contexts. We believe that the European version of 'known and acceptable' practice in Applied Learning is not immediately transferable into the local indigenous cultures of the ASEAN region, because this transfer of 'knowledge' is intimately bound with cultural assumptions. For us, knowledge, in relation to occupational standards, inclusivity and quality frameworks, is constructed and firmly entrenched in our western dominant ideologies.

This notion is illustrated in 'common sense' statements such as:

- Employer inputs into defining standards, competencies or learning outcomes are always best;

- Industry-led occupational standards are appropriate for Vocational Education and Training systems' development;

- Australian versions of 'working collaboratively' are appropriate in an Indonesian setting;

- Training is best instituted as a nationally uniform system;

- 'Guru-centered' learning is desirable in an Indonesian training context;

- Formally credentialed training is always a better use of available resources than informal training; and

- Vocational Skills Training

It is clear that some of the foregoing 'of course' statements are heavily ideological. Therefore if they are unthinkingly accepted as being based on universal 'commonsense' realizations, then the assumptions that underpin program design will be implemented without checking the authenticity that underpin these statements. In this circumstance, the complexities and incongruities associated with these realizations will begin to emerge.

In The Malang Declaration, Fairman et al. (2020) deconstructed the term 'industry expert' and, in doing so, revealed some of the underlying commonsense interpretations of the value associated with this type of language, and the consequent implications which arise when accepting without question these realizations (Fairman et al., 2020). The authors cautioned that when applying 'language' and 'practice', which is relevant in their own cultural context to other contexts, dominant values and ideologies permeate the mix. The authors argue that the onus is on the 'expert' to make clear these assumptions. Applied learning interventions currently operate at this ideological/mythological level, and we are concerned that we need to recognize, question and confront many hidden assumptions. Failure to recognize this need, and to consequently apply training in an unfamiliar environment, leads to what we consider to be 'vocational imperialism', suggesting that unexpected, unhelpful and possibly unjust outcomes can subsequently emerge.

We are therefore concerned that, too often, well-meaning technical experts from a Western nation can enter a recipient country offering aid, and the recipient communities accept this offer and await further 'western' assistance unquestioningly. This system was observed most clearly during the World War II in Papua and the South Pacific area. In a phenomenon described as the 'Cargo Cult', Melanesians cut down forests in order to create landing strips for the gift-bearing 'big birds'. That they never came again after allied operations ended in the Pacific (Jebens, 2004), is a poignant reminder of how well-meant actions can sometimes be misinterpreted. This example reminds us that visiting technical experts need to be acutely mindful of the impact of extolling the virtues of 'western' knowhow in an unfamiliar milieu without implementing a parallel program designed to respect and engage local issues, thus create meaningful and long-term experiences in a local context.

Voak and Fairman (2020) noted that foreign donors often externalize and reinforce their dominant cultural values (Voak \& Fairman, 2020). These authors make specific reference to vocational skills development in the non-formal education milieu in Timor-Leste. Boughton (2009) in Cooper and Walter's Learning/Work (Cooper \& Walters, 2009), argues that international experts and advisors, whilst expert in their respective fields, come from, or operate in, an unfamiliar ideological or mythological framework:

While the advice may appear to come from neutral well-qualified 'experts', it also appears to reflect the 
dominant thinking in many countries about the connection between training and employment, including the highly-contestable view that training, of itself, leads to lower unemployment (Cooper \& Walters, 2009 , p. 80).

We thus propose that Vocational Skills development interventions which are carried out by international presenters, need to be applied in a situation informed by a critical framework, which is often termed to be a 'semiotic' approach (Fairman, 2018). Development of such an intervention could begin by posing the following presumption: What is NOT included is as equally valid as what IS included. In this way, critical analysis of: (i) program and project designs, (ii) training needs assessments, (iii) occupational standards, (iv) evaluation and project reports, (v) recommendations and lessons learnt, and (vi) stakeholder involvement and engagement, all need to be instituted. Indeed, the entire plethora of conceived Vocational Program interventions would benefit by posing more generalised statements such as:

- It is not only what vocational training models are imported that matters, it is also what training models are NOT imported that matters;

- It is not what is in the policy that matters, it is also what is NOT in the policy that matters;

- It is not who is included in the strategic partnership that matters, it is also who is NOT included that matters;

- It is not what occupational standard is selected for industry inclusion, it is also what occupational standard is NOT selected for inclusion that matters;

- It is not which industry is selected for VET development, it is also which industry is NOT included that matters;

- It is not which participants are included, as much as which participants are NOT included; and

- It is not what IS, as much as it is also what is NOT.

The identification of what IS a result of intervention programs should be balanced by an identification of what does NOT emerge from those interventions. We argue that every person involved in the development of vocational interventions needs to examine their assumptions and share any concerns regarding the 'missing and hidden' elements. Suggestions of how these elements might be rectified to produce a more complete image is a universally shared responsibility. Notwithstanding this objective, we are keenly conscious of Hall's views, that it is not what IS in an image so much as what is NOT in the image that tells 'us' more about ourselves. It is this issue that should be squarely faced by international practitioners. Given that this reflection includes our notions of our constructed world or our 'common sense truths' about ourselves (Hall, 1983), and that the impact of this 'constructed world' represents an example of 'ideology at work', the reflective approach provides a rich metaphor that could assist practitioners in developing more meaningful practice interventions. We assert here that deconstructing these 'common sense truths' in the Vocational Education and Training development context, is critical to gaining an understanding of the unintended nature, extent and depth of social control and vocational imperialism. Attention to this concern will provide us with a greater understanding of, and clarity about, what is being offered and what is being implemented (Shahjahan et al., 2015).

We have argued here that by applying this deconstruction approach, hitherto alternate questions about the intervention processes can be posed. On this basis, we suggest that such an enlightened critical theorist approach will be made easier if an environment exists which allows the open posing of these questions and the seeking of practical answers. Unfortunately however, this environment currently does not exist, as practitioners are more likely to attempt to respond directly to the key performance indicators embedded in the project provisions and contractual agreements. We are convinced that it is even more challenging in instances where practitioners have personal vested interests in continuing to engage with these communities without critically reflecting upon their practice.

\section{Meaningful Practice}

It is against this reflective background that this paper proposes a model known as the 'meaningful practice intervention' (Fairman, 2018). This approach will be suitable to assist practitioners, recipients and donors who wish to carefully explore the rationale and approach applied in individual Vocational Training interventions. Issues under investigation relate to the design, implementation and evaluation phases of their current interventions, and would provide a basis for this critical reflection practice. Furthermore, Fairman (2018) proposes a practice model which could guide practitioners in developing practice interventions in applied learning environments in the ASEAN region. This model was employed in the development of occupational standards for the supply chain and logistics industry in Indonesia and could have wider application in other settings. Apropos of the recognition of engaging 'local' stakeholder and industry input into the consequential design (Abdullah, 2014), this 'framework' of reflective practice may have broader application in the up-skilling of local training providers.

The importance of proposing a scheme of meaningful practice interventions that may improve external 'engagement' in Indonesia, in which we openly, and respectfully, recognize and acknowledge local ownership and 
contributions. We are cognisant that in an Indonesian cultural milieu of many varied and divergent sub-cultures, that there is not one approach that meets all the needs of this society (Bayu Adi et al., 2018). Imported interventions need to be correlated and harmonized with the specific and localized requirements for that intervention (Gentile, 2019).

Fairman's model (2018), which guides the practitioner toward meaningful practice outcomes, requires that the 'practitioner' also applies this framework of ethical behavior, stated intentions, recipient country considerations, local considerations, evaluation practice and sharing with future design interventions. The model is expressed in Figure 1.

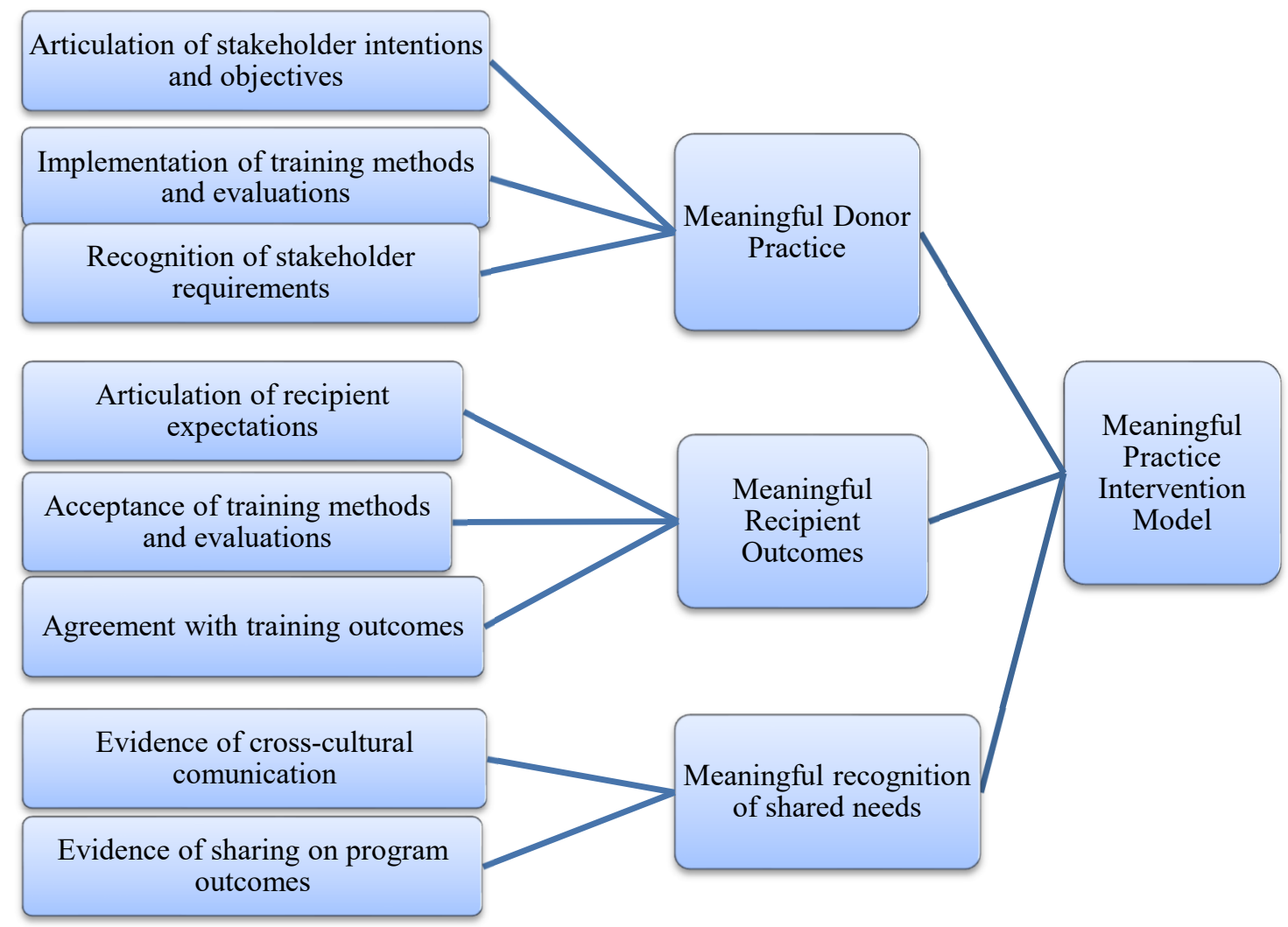

Figure 1. Meaningful Practice Intervention Model (Fairman, 2018).

This model reviews the critical phases required to align practice elements of evidence-based interventions (Farmer, 2020) covering key issues of ethical behavior, intentions, recipient country consideration, local consideration, evaluation practice and sharing of program outcomes. In this paper we have argued that in many development contexts a 'lens' through which we can ask difficult and purposefully designed questions around the intent of our development practice is required and this 'Model' provides that structure, as illustrated by the following questions: (i) What are the intentions behind stakeholder engagement?; (ii) What is the evidence of cross-cultural engagement and communication?; (iii) Where is the evidence of sharing program outcomes?; (iv) Where is the recognition of stakeholder requirements?

In applying this 'Model', we acknowledge that a more systematic examination of the motives, rationale and underlying principles are examined and made transparent. Whilst the government, donors and industry may seek to assert their own 'individual perspectives' on program implementation, the onus is also upon the designers, managers and implementers to explore their own underlying values and assumptions. The purpose of this model is to provide practitioners, government and donors with a common, tested framework for examining program design, implementation and evaluation in line with the six key ideas/principles of; ethical behavior, intentions and objectives, recipient country consideration, consideration of local input, acceptance of training interventions, and sharing of outcomes. It is in this context that respectful practice and positive outcomes are examined.

\subsection{Ethical behavior}

Meaningful practice requires an examination of the ethical behavior associated with motivations behind actions, the choices, the decisions and the rationale behind the designed intervention. Ethical behavior begins by reviewing the motivations behind the actions, asking Why is one action taken rather than another. The choices involved in determining the approach could best be examined by asking questions such as; What was the rationale for the intervention? What alternatives exist for achieving the same outcomes? How were the decisions made to determine 
the planned intervention? Who was involved in determining the planned intervention? Who determined what the planned outcomes would be? How were these outcomes described and determined? What basis exists for determining the 'Local' engagement in the activity? What justification exists for this program? We suggest that these reflective questions provide a good practical basis to develop a more inclusive, and less ideological, intervention.

\subsection{Intentions and objectives}

Describing and detailing the anticipated 'objectives' will allow practitioners to evaluate and measure the progress toward program outcomes, and determining these objectives. Developing respectful practice and positive outcomes may require asking difficult and challenging questions of all stakeholders. Further to defining intentions and objectives practitioners, governments and donors need to establish evaluation frameworks at the beginning of interventions, and proceed against the background of the designed objectives, ensuring that there are accurate and agreed measures of these objectives which are available. The importance of clearly stating, and agreeing on, intentions and objectives of a program cannot be underestimated.

\subsection{Recipient country consideration}

Recipient countries that develop human capability development plans are able to identify policy imperatives and training priorities through these plans, and this will assist donors and practitioners to match their training offerings with these plans and within an recipient country institutional context. When donors are working with Ministries, this requires detailed examinations of a recipient country's expectations for each Ministry. The difficulty in gaining uniformity around recipient country consideration, involves teacher/trainer competencies, which suggests that a more uniform approach to competency development is required in Indonesia (Sayuti, 2016). Training needs assessments will provide an opportunity to adjust program interventions to meet these recipient country circumstances.

\subsection{Consideration of stakeholders and local input}

There is a central requirement of building trust in training projects when local training institutes are 'partnered' with industry and these need to be explored as to the requirements to achieve sustainability. Adopting an 'inprinciple' position of seeking 'local' training institution input, places a transparent priority on working with locals. Working with local 'institutes' creates situations, where the 'ultimate beneficiaries' of Vocational Training interventions are easily identified. Fairman (2018) describe the critical initial engagement between trainer and industry, together with the training needs assessments which have been carried out by VET practitioners (Fairman, 2018). These issues are required in order to present appropriately designed training experiences, presented in innovative and customised ways, which are geared to achieve specific learning outcomes for the industry.

\subsection{Acceptance of 'cross-cultural' practice}

There are many challenges associated with working 'collaboratively' with foreign facilitators, and also to the importance of using a 'lens' of 'cross-cultural' understandings to interpret behavior and actions. The acceptance of 'cross-cultural' practice is a requirement for sustainability in the Vocational Education and Training sector. Cross cultural communication skills are essential for partnership engagement, particularly when moving across cultures within institutions, across organisations and national borders, and within culturally diverse countries.

\subsection{Sharing program outcomes}

We have emphasized in this paper that it is important to develop and explore diverse ways of sharing knowledge, skills and attitudes. As a practical exemplar for such an approach, we have described the challenges of working collaboratively in developing the 'Malang Declaration'. In this discussion, there was a tension between the required industry occupational standards, the perceptions of the Industry Reference group, the many recognized challenges of working with a variety of industries and the ingrained approaches of the 'Bule'. However, it was observed that by being transparent and sharing program intentions, these actions worked to bring organizations and people together and resulted in improved outcomes.

Whilst the Meaningful Practice Intervention Model approach goes some way to 'unpacking' these interventions and questioning the hidden assumptions, it by no means covers all of the possible manifestations of these interventions. With this caveat, the purpose of these reflections was to provide practitioners with a framework for dispassionately examining their program decisions, and thus advocating for trustworthy modifications to their practice.

\section{Concluding remarks}

Vocational Imperialism exists when we accept the 'common sense truths' of the way things ought to be without questioning their legitimacy. We have argued that a transformative learning process involves transforming frames 
of reference through critical reflection, an evaluation of contested beliefs through dialogue, the taking of action about one's reflective insight, and critically assessing the action taken (Babacan \& Babacan, 2018, 2015). This paper has sought to confront the language of 'of course', and we recognize the ease with which practitioners continue to engage in the 'way things are' without challenging the circumstances that instigate this belief. The 'business as usual' approach is under challenge, and we have posed difficult and confronting questions around existing 'institutionalized practice'. There is a need to confront existing practices/systems in order to improve outcomes and challenge the perpetuation of 'dominant values'. Despite the difficulty and personal challenges involved posing questions of developed program implementation and designed 'interventions', if these questions remain 'unanswered' then dominant values are likely to endure.

Constraints currently exist in the Vocational Education and Training development context, which could seriously limit the sector's ability to change. These constraints include the practical issue that these development programs are often 'short' in duration, which mitigates against detailed reflection opportunities. It has been argued that engaging in a balanced cross-culturally relevant training approach requires application of an appropriate model of planned intervention. This paper suggested the possible introduction of a regional 'model of meaningful practice intervention' (Fairman, 2018), which was specifically developed to guide future practice, and which includes suggestions and approaches to give voice to the recipients of VET interventions. Its use will avoid the growing charge of vocational imperialism or the focus on 'outsider' perceptions, which are increasingly proving to be of questionable validity. We suggest that further research into the application of the model examined in this paper would provide valuable insights for policy makers, administrators, and trainers and assessors in government and industry in Indonesia and beyond.

\section{References}

Abdullah, H. (2014). The role of VET providers in training partnerships with industry in East Java, Indonesia. TVET@,Asia, Issue1,1-14.

Allais, S. (2014). Selling Out Education National Qualifications Frameworks and the Neglect of Knowledge.

Alphonse, M., George, P., \& Moffatt, K. (2008). Redefining social work standards in the context of globalization: Lessons from India. International Social Work, 51(2), 145-158.

Babacan, A., \& Babacan, H. (2015). A transformative approach to work integrated learning in legal education. Education + Training.

Babacan, A., \& Babacan, H. (2018). Repositioning lifelong learning in legal education. Widening Participation and Lifelong Learning, 20(2), 122-148.

Barron, B., \& Darling-Hammond, L. (2008). Teaching for Meaningful Learning: A Review of Research on InquiryBased and Cooperative Learning. Book Excerpt. George Lucas Educational Foundation.

Bayu Adi, L., Supriyono, \& Sri, W. (2018). An investigation of local wisdom to support adult literacy program. PEOPLE: International Journal of Social Sciences, $4(2)$. https://grdspublishing.org/index.php/people/article/view/1559

Beck, U. (2000). The cosmopolitan perspective: sociology of the second age of modernity. The British journal of sociology, 51(1), 79-105.

Cooper, L., \& Walters, S. (2009). Learning/work: Turning work and lifelong learning inside out. http://repository.hsrc.ac.za/handle/20.500.11910/4664

Dayaram, K., Lambey, L., Burgess, J., \& Afrianty, T. W. (2020). Developing the Workforce in an Emerging Economy: The Case of Indonesia. Routledge.

Deutsch, M. (2006). A framework for thinking about oppression and its change. Social Justice Research, 19(1), 7 41.

Fairman, B. (2018). Looking for a way out: Skills development and training and its impact on aid practices and their development outcomes, with particular reference to Indonesia and Timor-Leste Victoria University].

Fairman, B., Voak, A., Abdullah, H., \& Amaripuja, I. (2020). The 'Malang Declaration': Models of Engagement within Research Programs. KnE Social Sciences, 4(12). https://doi.org/10.18502/kss.v4i12.7580

Farmer, T. W. (2020). Reforming research to support culturally and ecologically responsive and developmentally meaningful practice in schools. Educational Psychologist, 55(1), 32-39.

Farmer, T. W., Gatzke-Kopp, L., \& Latendresse, S. J. (2020). The development, prevention, and treatment of emotional and behavioral disorders: An interdisciplinary developmental systems perspective. In Handbook of Research on Emotional and Behavioral Disorders (pp. 3-22). Routledge.

Freire, P. (1970). Pedagogy of the oppressed. Continuum. New York.

Freire, P., \& Shor, I. (1986). A Pedagogy for liberation: Dialogues on transforming education south hadley. $M A$ : Bergin and Garvey.

Gale, T., \& Densmore, K. (2000). Just schooling. Open University Press Buckingham.

Gentile, E. (2019). Skilled Labor Mobility and Migration: Challenges and Opportunities for the ASEAN Economic Community. Edward Elgar Publishing. 
Gopalkrishnan, N., \& Babacan, H. (2015). Cultural diversity and mental health. Australasian Psychiatry, 23(6 suppl), 6-8.

Hall, S. (1983). Ideology in the Modern World. In M. Robertson (Ed.), (pp. 68). Melbourne: Latrobe University.

Hallinger, P. (1998). Educational change in Southeast Asia: The challenge of creating learning systems. Journal of Educational Administration.

Hines, A., Cohen, E., Nguyễn, H. T., Drabble, L., Han, M., Sen, S., \& Trâàn, T. Đ. (2015). Improving social work education in Vietnam through international cooperation: The 'Social work education enhancement program'. Social Work Education, 34(6), 716-728.

Jebens, H. (2004). Cargo, cult, and culture critique. University of Hawaii Press Honolulu.

Lawler, A., \& Sillitoe, J. (2013). Facilitating 'organisational learning'in a 'learning institution'. Journal of Higher Education Policy and Management, 35(5), 495-500.

McIntosh, P. (2015). Extending the knapsack: Using the white privilege analysis to examine conferred advantage and disadvantage. Women \& Therapy, 38(3-4), 232-245.

Mezirow, J. (2000). Learning as Transformation: Critical Perspectives on a Theory in Progress. The Jossey-Bass Higher and Adult Education Series. ERIC.

Neumann, L. (2021, 27/07/2021). The Future of Education in Indonesia In Envisioning Indonesia's Future With Education Minister Nadiem Makarim. https://open.spotify.com/episode/1czRSk2Uphi5dGtlzkNigt?si=VKoKkIPHQhOYDMUaFSQ\&dl_branch $=1 \&$ nd $=1$

Olaniran, B. A., \& Agnello, M. F. (2008). Globalization, educational hegemony, and higher education. Multicultural Education \& Technology Journal.

Robinson, W. I., William, I., \& Robinson, W. I. (2004). A theory of global capitalism: Production, class, and state in a transnational world. JHU Press.

Sayuti, M. (2016). The Indonesian Competency Standards in Technical and Vocational Education and Training: an Evaluation of Policy Implementation in Yogyakarta Province Indonesia The University of Newcastle Australia].

Stanchfield, J. (2013). What is experiential education. Retrieved from http://www.experientialtools.com/about/experiential-education.

Voak, A., \& Fairman, B. (2020). Crossing Borders: Anticipated Challenges in Building a Body of Knowledge for the Digital Printing Sector to Promote Relevant Human Capability and Mobility in a Local Context. Journal of Printing Science and Technology, 57(1), 29-32. 